\title{
Autismo associado à epilepsia: relato de caso
}

\author{
Autism associated with epilepsy: case report
}

Autismo asociado con la epilepsia: reporte de caso

Caroline Cunha da Rocha ${ }^{1 *}$, Cristine Bessa Gondim², Tayana Aguiar Gomes², Luciana Cristina Menezes Martins dos Santos ${ }^{1}$, Ivy de Almeida Cavalcante e Silva ${ }^{3}$.

\section{RESUMO}

Introdução: O autismo é uma doença do desenvolvimento, onde são notados problemas quanto à interação social, capacidade de comunicação e movimentos repetitivos. Pode variar desde formas graves de deficiência mental até pessoas com inteligência normal e vida independente. A associação desta entidade nosológica com a epilepsia é pouco documentada na literatura e estudos mostram que a prevalência de epilepsia chega a ser de 1 a $2 \%$ maior nos pacientes com autismo em relação à população em geral. As condutas escolhidas para tratar este paciente com tais doenças devem incluir uma equipe multidisciplinar, com finalidade de uma melhor qualidade de vida e atividades que integradoras para o paciente, indo desde a uso de fármacos à terapia ocupacional, fisioterapia e outras atividades. Detalhamento do caso: paciente, sexo feminino, 8 anos e 9 meses, desde os 6 meses de idade, apresentava atraso no desenvolvimento, recebeu diagnostico de Transtorno do Espectro Autista (TEA) associado à epilepsia atendida em serviço de referência em pediatria em Belém-Pará e tratada inicialmente com $\mathrm{Fe}_{2} \mathrm{SO}_{4}$, carbamazepina e risperidona. Conclusão: Poucos são os relatos na literatura de associação entre o transtorno do espectro autista (TEA) e epilepsia na literatura, portanto é importante estudar o tema, já que a prevalência de epilepsia é maior nos pacientes que possuem TEA. O profissional que atende o paciente com TEA deve atentar para a investigação da epilepsia, não devendo negligenciar uma possível associação entre as patologias, mas sim oferecer um diagnóstico precoce, tratamento multiprofissional e uma melhor qualidade de vida para a criança.

Palavras-chave: Epilepsia, Autismo, Transtorno do espectro autista, Atraso no desenvolvimento.

\begin{abstract}
Introduction: Autism is a developmental disorder, where problems are noted regarding social interaction, communication skills and repetitive movements. It can range from severe forms of mental deficiency to people with normal intelligence and independent living. The association of this nosological entity with epilepsy is poorly documented in the literature and studies show that the prevalence of epilepsy reaches 1 to $2 \%$ higher in patients with autism in relation to the general population. The pipelines chosen to treat this patient with such diseases should include a multidisciplinary team, aiming at a better quality of life and integrating activities for the patient, ranging from the use of drugs to occupational therapy, physiotherapy and other activities. Case report: patient, female, 8 years and 9 months, from 6 months of age, presented developmental delays, received diagnosis of Autism Spectrum Disorder (ASD) associated with epilepsy attended at a referral service in pediatrics in Belém- Pará and initially treated with $\mathrm{Fe}_{2} \mathrm{SO}_{4}$, carbamazepine and risperidone. Conclusion: There are few reports in the literature about the association between autism spectrum disorder (ASD) and epilepsy in the literature, so it is important to study the topic, since the prevalence of epilepsy is higher in patients with ASD. The professional who attends the patient with ASD should pay attention to the investigation of epilepsy, not neglecting a possible association between the pathologies, but rather offer an early diagnosis, multiprofessional treatment and a better quality of life for the child.
\end{abstract}

Keywords: Epilepsy, Autism, Autism spectrum disorder, Development.

\footnotetext{
${ }^{1}$ Pronto Socorro Municipal de Belém - Mario Pinotti, Belém-PA. * E-mail: carolinegatti@gmail.com

2 Universidade do Estado do Pará, Belém-PA.

${ }^{3}$ Fundação Santa Casa de Misericórdia do Pará, Belém-PA.
} 


\section{RESUMEN}

Introducción: el autismo es un trastorno del desarrollo, donde se observan problemas relacionados con la interacción social, las habilidades de comunicación y los movimientos repetitivos. Puede ir desde formas severas de deficiencia mental hasta personas con inteligencia normal y vida independiente. La asociación de esta entidad nosológica con la epilepsia está poco documentada en la literatura y los estudios muestran que la prevalencia de la epilepsia alcanza de 1 a $2 \%$ más en pacientes con autismo en relación con la población general. Las tuberías elegidas para tratar a este paciente con tales enfermedades deben incluir un equipo multidisciplinario, que apunte a una mejor calidad de vida e integre actividades para el paciente, desde el uso de medicamentos hasta la terapia ocupacional, la fisioterapia y otras actividades. Reporte de caso: paciente, mujer, 8 años y 9 meses, a partir de los 6 meses de edad, presentó retrasos en el desarrollo, recibió un diagnóstico de trastorno del espectro autista (TEA) asociado con epilepsia atendida en un servicio de referencia en pediatría en Belém-Pará y fue tratada inicialmente con $\mathrm{Fe}_{2} \mathrm{SO}_{4}$, carbamazepina y risperidona. Conclusión: Existen pocos informes en la literatura sobre la asociación entre el trastorno del espectro autista (TEA) y la epilepsia en la literatura, por lo que es importante estudiar el tema, ya que la prevalencia de epilepsia es mayor en los pacientes con TEA. El profesional que atiende al paciente con TEA debe prestar atención a la investigación de la epilepsia, sin descuidar una posible asociación entre las patologías, sino ofrecer un diagnóstico temprano, un tratamiento multiprofesional y una mejor calidad de vida para el niño.

Palabras clave: Epilepsia, Autismo, Desorden del espectro autista, Desarrollo.

\section{INTRODUÇÃO}

O transtorno do Espectro Autista (TEA) é um distúrbio do desenvolvimento neurológico, de início precoce, que causa dificuldades na interação social e comunicação; movimentos estereotipados podem estar presentes. O fenótipo do paciente com TEA pode variar enormemente, fazendo parte do espectro, tanto pacientes com deficiência intelectual (DI) grave como pessoas com quociente de inteligência (QI) normal e vida independente. Autistas podem apresentar algumas comorbidades associadas, tais como: Transtorno do Déficit de Atenção e hiperatividade (TDAH), distúrbios do sono, alterações gastrointestinais e epilepsia (APA; 2013; CHRISTENSEN et al, 2016).

O TEA acomete cerca de $1 \%$ da população. Algumas literaturas sugerem prevalência de 62 casos para cada 10.000 pessoas. É quatro vezes mais frequente no sexo masculino que feminino (4:1) e seu risco de recorrência entre irmãos é de $3-5 \%$, sugerindo uma predisposição genética para sua ocorrência (CHRISTENSEN et al, 2016; FONBONNE, 2009). Já a prevalência de epilepsia é de 70\% entre os transtornos desintegrativos da infância. No entanto, há carência de estudos clínicos sobre a associação do TEA com a epilepsia. Estima-se que os pacientes com este transtorno possuam um risco 1 a $2 \%$ maior de apresentar epilepsia em comparação à população geral, caracterizando assim o TEA como fator de risco para surgimento de epilepsia (CANITANO, 2007; SPENCE E SCHNEIDER, 2009).

A epilepsia paroxística é caracterizada por uma atividade cerebral anormal associada a várias alterações de conduta e, tal qual os transtornos do desenvolvimento, não é uma entidade patológica isolada, podendo acompanhar o TEA. A frequência de epilepsia em pacientes com TEA aumenta em menores de 5 anos e principalmente em adolescentes. Existe uma ampla variação entre os tipos e a prevalência de crises epilépticas; qualquer modalidade desta patologia pode estar associada ao TEA. Alguns estudos relatam que existe uma maior probabilidade de ocorrência de crises epilépticas em pacientes com retardo mental grave associado (CANITANO, 2007; SPENCE E SCHNEIDER, 2009).

Foram observadas algumas alterações eletroencefalográficas nos pacientes com TEA, tais quais, alterações epileptiformes e também outras inespecíficas, como assimetria e alentecimento. Porém o Eletroencéfalograma (EEG) é solicitado somente se o paciente apresentar alguma suspeita clínica de epilepsia (SPENCE E SCHNEIDER, 2009). O diagnóstico de TEA é essencialmente clínico e baseia-se nos critérios apresentados no Manual Diagnóstico e Estatístico de Transtornos Mentais (DSM-5) e pelo CID-10 (Classificação Internacional de Doenças da OMS) para autismo. Os sintomas costumam estar presentes antes dos 3 anos de idade e sendo possível fazer diagnóstico com 18 meses de idade (AAP, 2014). 
Existem ainda alguns instrumentos diagnósticos utilizados na avaliação do TEA, como Childhood Autism Rating Scale - CARS (Escala de avaliação da gravidade para autismo infantil), Escala d'Avaluació dels Trests Autistes, ATA (Escala de avaliação de traços autistas), Checklist for Autism in Toddlers, CHAT (Escala para rastreamento de autismo em crianças com até 3 anos), entre outros (BRASIL 2018).

Após o diagnóstico, um dos objetivos fundamentais do atendimento aos indivíduos com TEA é o de habilitálo a participar de modo ativo e independente das atividades que lhes são apresentadas, com uma intervenção multidisciplinar, envolvendo técnicas de mudança de comportamento, programas educacionais ou de trabalho e terapias de linguagem/comunicação. Os objetivos são ampliar os ganhos funcionais, auxiliar a busca pela realização independente das atividades de vida diária e social (AVDS), melhorando assim sua qualidade de vida. Para isto, é essencial trabalhar com uma equipe multiprofissional bem treinada em análise comportamental funcional e em técnicas de mudança de comportamento (GADIA et al, 2004; BRASIL, 2015).

Concomitantemente, a farmacoterapia é instrumento terapêutico importante, através da utilização de antipsicóticos que conseguem controlar os "sintomas alvo" como as condutas agressivas e autolesivas, episódios de raiva e descontrole, as dificuldades para conciliar o sono, a inquietude extrema, além de algumas estereotipias motoras ou comportamentos repetitivos que podem ser atenuados. Tais medicamentos não atuam no cerne da doença e sim em seus sintomas, em especial, naqueles que mais prejudicam a qualidade de vida destes pacientes (BRASIL, 2015).

\section{DETALHAMENTO DO CASO}

L.C.C., sexo feminino, 8 anos e 9 meses, desde os 6 meses de idade a menor apresentava atraso no desenvolvimento, sendo evidenciado por não alcançar os marcos do desenvolvimento para sua idade. Segundo a mãe, algumas vezes apresentou quadros convulsivos. No momento da primeira consulta, relato de dificuldade de aprendizado e déficit de atenção.

Em seu histórico familiar não apresenta casos iguais ao seu ou de outras patologias, porém, possui pais consanguíneos. Mãe realizou 4 consultas de pré-natal, realizou sorologia para TORCHS com sorologias não reagentes e não há relato de patologias e intercorrências durante a gravidez. A menor nasceu via parto vaginal a termo, apresentou cianose e anóxia, sem outras intercorrências.

Em abril de 2012, evoluía bem de saúde, apenas apresentando anemia. Ao teste de Denver II - alterado para linguagem, motor grosseiro, motor adaptativo e pessoal. Teve como hipóteses diagnósticas: desenvolvimento - atraso de desenvolvimento e atraso de linguagem; etiológico - anóxia perinatal interrogada; clínico - encefalopatia crônica não evolutiva (ECNE). Solicitados exames complementares (hemograma e dosagem de cloro e sódio no suor); introdução da medicação $\mathrm{Fe}_{2} \mathrm{SO}_{4}$; encaminhamentos para serviço social, enfermagem, otorrinolaringologista, oftalmologista, neurologista, fisioterapia, terapia ocupacional, fonoaudiologia; retorno. Ainda em abril de 2012, em atendimento com assistente social, foi evidenciado ótimo relacionamento familiar e o desejo de melhora do quadro de saúde da menor esperado pelos pais da paciente.

Em seu retorno no mês de julho de 2012, apresentou como intercorrências apenas quadro de tosse e secreção ocular com hiperemia. A avaliação de seu desenvolvimento mostrou: atrasos em seu desenvolvimento motor grosseiro, motor fino, linguagem, pessoal/social, problema de comportamento, evidenciado por isolamento. Não apresentou convulsão entre as consultas médicas. Ainda não estava frequentando as reabilitações indicadas. Ao exame físico, foi encontrada hipotonia do pescoço e roncos difusos no hemitórax esquerdo. Quanto aos exames complementares: hemograma de abril de 2012 mostrou $\mathrm{Hb}: 10.9 \mathrm{~g} / \mathrm{dL}$; Ht: 34\%; leucócitos: 10.100/mm³; eosinófilos:11\%. Foi realizada tomografia de crânio em maio de 2012, sendo encontrada mega cisterna magna. Estava eutrófica, com alimentação adequada, vacinas atualizadas, apenas havendo quadro de infecção de vias aéreas superiores. Como conduta, foram prescritos mebendazol e $\mathrm{Fe}_{2} \mathrm{SO}_{4}$, e marcado retorno.

Consulta de retorno em setembro de 2013, queixa de tumoração e feridas no ouvido, com quadro iniciado cerca de 8 dias antes da consulta, com presença de sinais flogísticos em toda região auricular esquerda da 
menor, com presença de secreção hematopurulenta que possuía odor fétido, sendo medicada com amoxacilina e pomada de neomicina. Além disso apresentava autoagressão quando estava estressada e diminuição de sensibilidade dolorosa e térmica; uma internação hospitalar de 3 dias por bronquite, com melhora do quadro. Quanto ao seu desenvolvimento: normalização do desenvolvimento motor grosseiro, mas os demais parâmetros analisados ainda continuavam alterados, sendo que em relação ao seu comportamento, estava apresentando agressividade e problemas de aprendizagem. Não apresentou convulsão entre as consultas. Recebeu alta do serviço de fisioterapia, sem realizar outras reabilitações.

Exame físico com alteração apenas em pavilhões auriculares. Quanto aos exames complementares: em julho de 2012 foi feita avaliação auditiva infantil, mostrando emissões otoacústicas evocadas e produto de distorção presente bilateralmente; em setembro de 2012, foi realizada a pesquisa de cloreto no suor, porém, a quantidade de suor foi insuficiente para a análise. Em outubro de 2012, foi realizada pesquisa de potencial auditivo evocado de tronco encefálico com o seguinte resultado: presença de ondas III e V de Jewett a 80 dBNA em OD; presença de ondas I, III e V de Jewett a 80 dBNA em OE; latência absoluta e interpicos dentro da normalidade em AO; limiar eletrofisiológico para clicks de 40 dBNA em OD e OE.

Os diagnósticos nutricional, alimentar e vacinal mantiveram os mesmos da última consulta. Já o diagnóstico clínico, devido à intercorrência, foi de otite interrogada. Diagnóstico do desenvolvimento funcional foi de atraso de linguagem, alteração comportamental e de perda de sensibilidade térmica e dolorosa. $O$ etiológico foi de anóxia perinatal interrogada e de consanguinidade. O diagnóstico clínico do desenvolvimento foi de encefalopatia crônica não evolutiva e distúrbio sensorial. Foi feito encaminhamento para genética.

Em junho de 2014, foi aplicado um questionário com critérios diagnósticos para o transtorno autista, havendo pontuação total de itens igual a 8 , distribuídos como 4 pontos no critério 1 (comprometimento qualitativo da interação social); 2 pontos no critério 2 (comprometimento qualitativo de comunicação); 2 pontos no critério 3 (padrões restritos e repetitivos de comportamento, interesses e atividades). Estes critérios diagnósticos foram novamente avaliados em abril de 2015, havendo pontuação total de itens igual a 14 , pontuando todos os campos nos critérios 1 e 2 , e no critério 3 , com exceção do item preocupação persistente com partes de objetos, pontuou todos os demais do referido critério, havendo assim diagnóstico de autismo.

Em consulta de retorno em agosto de 2015, foi diagnosticada com epilepsia, tendo apresentado crises do tipo tônico-clônica generalizada entre as consultas e nos dias que a antecederam, sendo introduzida carbamazepina $5 \mathrm{~mL}, 1 \mathrm{vez}$ ao dia. Fez uso de risperidona. Em dezembro de 2015, consulta de retorno já com o diagnóstico de transtorno autista. Apresentava menor agressividade com o uso da medicação prescrita anteriormente, sem demais alterações. Seu desenvolvimento continuava atrasado em todos os parâmetros. Estava sem atividades de reabilitação. Exame físico sem alterações. Diagnósticos nutricional, clínico e vacinal dentro dos padrões normais. Quanto ao seu diagnóstico do desenvolvimento, apresentava alteração de comportamento, linguagem e interação social, por etiologia multifatorial e diagnóstico clínico de autismo. Consulta de retorno como conduta.

Em agosto de 2016, evoluía com progresso na parte motora, porém agressividade persistia e sono estava preservado, com intercorrência apenas de infecção de vias aéreas superiores (IVAS), sem convulsões entre as consultas. Sem atividades de reabilitação. Ao exame físico, apenas estrabismo e abdome globoso, porém sem visceromegalias ou massas palpáveis. Diagnósticos nutricional, clínico e vacinal sem alterações em relação a última consulta. Quanto ao desenvolvimento: funcional - alteração de comportamento, linguagem e interação social; etiológico - fator de risco pré-natal, perinatal; clínico - autismo. Exames solicitados parasitológico de fezes e pesquisa de sangue oculto nas fezes. Orientações para retorno de ações reabilitadoras e alta do ambulatório de desenvolvimento e manter acompanhamento no ambulatório de autismo.

\section{DISCUSSÃO}

Estudos mostram que a prevalência de epilepsia é cerca de $1 \mathrm{a} 2 \%$ mais alta nos pacientes com transtorno do espectro autista (TEA) em comparação com a população em geral, sendo assim, um fator de risco para o 
desenvolvimento de epilepsia (KUCZYNSKI, 2016). O transtorno do espectro autista (TEA) se enquadra nos transtornos de desenvolvimento, sendo um transtorno mental da infância e quanto mais precoce for a idade de aparecimento dos sintomas, maior será o risco de que a doença seja crônica (BRASIL, 2015). A maior porcentagem de epilepsia associada ao TEA é no gênero feminino (MUÑOZ-YUNTA et al, 2006). A paciente aqui estudada teve início de sintomas percebidos pela mãe antes de 1 ano de idade, sendo desde então observados atraso em seu desenvolvimento, evoluindo para doença crônica, porém, seu diagnóstico de transtorno do espectro autista só foi feito aos 4 anos e 6 meses.

A epilepsia associada ao TEA é uma alteração bem documentada (KUCZYNSKI, 2016; MUÑOZ-YUNTA et al, 2006; MUÑOZ-YUNTA et al, 2003). Alguns autores acreditam que a maior ocorrência de epilepsia em pacientes autistas esteja relacionada com o grau de retardo cognitivo associado, não com a fisiopatologia do autismo por si só. Outros autores falam a favor de haver causas multifatoriais, como idade, grau de atividade do TEA. O diagnóstico de epilepsia da paciente foi feito aos 5 anos e 8 meses, após ter sido feito o diagnóstico de TEA. Está descrito na literatura que existem dois picos de idade onde a frequência da manifestação inicial da epilepsia no autismo é maior. O primeiro pico é até os 3 anos de idade e o segundo é durante a puberdade (MUÑOZ-YUNTA et al, 2006). Constatamos que a paciente teve seu diagnóstico feito para epilepsia em uma idade fora destes picos, porém, já havia manifestações de epilepsia desde os 2 anos e 7 meses, estando assim dentro do primeiro pico de frequência.

São descritos vários tipos de crises epilépticas em pacientes com TEA, como crises parciais complexas, espasmos infantis, crises mioclônicas, ausências atípicas, crises tônico-clônicas generalizadas e crises atônicas (MUÑOZ-YUNTA et al, 2006). Um estudo mostra que convulsões ocorrem em 16 a 35\% de crianças com TEA, sendo os maiores fatores de risco associados para o desenvolvimento de epilepsia são retardo mental severo, e a combinação de deficiência mental severa com déficit motor. Se déficits cognitivos e motores forem excluídos, o único outro fator associado a um risco aumentado de epilepsia em crianças com autismo é o tipo de déficit de linguagem (GADIA et al, 2009). Qualquer tipo de convulsão pode ocorrer em crianças autistas. A paciente apresentou crises epilépticas do tipo tônico-clônica generalizada. Além disso, a paciente apresentava atrasos de aprendizagem, comportamento e alterações motoras, podendo estes ser fatores de risco presentes para o desenvolver o quadro de autismo associado à epilepsia.

Estudos de neuroimagem mostram que uma heterogeneidade de acometimento dos pacientes com TEA, que podem ser malformações corticais, hipoplasia de tronco cerebral, alargamento de ventrículos laterais, entre outros achados. Porém, nenhuma dessas alterações chega a ser específica de autismo (GADIA et al, 2009). A neuroimagem obtida por tomografia de crânio da paciente deste caso evidenciou megacisterna magna que é uma alteração que pode cursar com quadro de epilepsia.

O uso de medicações voltadas para tratamento de TEA ainda é pouco. São usados neurolépticos, antipsicóticos atípicos, antidepressivos tricíclicos, inibidores seletivos da receptação de serotonina (ISRS), antagonista seletivo de monoaminas cerebrais, entre outras (GADIA et al, 2009). No caso da paciente deste estudo, foi administrada carbamazepina, que é um antidepressivo tricíclico com ações antiepilépticas, neurotrópicas e é um agente psicotrópico, e como a paciente apresentou epilepsia, esta droga foi escolhida. Também foi feita administração de risperidona, que é uma droga antagonista seletiva de monoaminas cerebrais, e tem seu uso no TEA para tratamento de irritabilidade, sintomas de agressividade, autoagressão em crianças e adolescentes com TEA, sendo que a paciente apresentou episódios de irritabilidade, agressividade e autoagressão, sendo uma droga bem indicada para o caso.

Vários trabalhos trazem o uso de ferramentas como análise genética, estudo eletroencefalográfico, ressonância nuclear magnética (RNM), magnetoencefalografia, tomografia por emissão de prótons (PET), estudos de rede de interação neuronal e análise de neurotransmissores para melhor conhecer a associação entre o TEA e a epilepsia, o que para a paciente deste relato de caso, ainda não foi possível o uso de tais recursos, porém, não sendo negligenciado um tratamento multiprofissional de qualidade para suas patologias.

Poucos são os relatos na literatura de associação entre o transtorno do espectro autista (TEA) e epilepsia na literatura, portanto é importante estudar o tema, já que a prevalência de epilepsia é maior nos pacientes 
que possuem TEA. Estudos como este acrescentam informações importantes para ajudar na ampliação conhecimento sobre as patologias associadas, fazendo com que haja melhor abordagem clínica e uma boa evolução no prognóstico do paciente. O profissional que atende o paciente com TEA deve atentar para a investigação da epilepsia, não devendo negligenciar uma possível associação entre as patologias, mas sim oferecer um diagnóstico precoce, tratamento multiprofissional e uma melhor qualidade de vida para a criança.

\section{REFERÊNCIAS}

1. AMERICAN PSYCHIATRIC ASSOCIATION (APA). Diagnostic and statistical manual of mental disorders. 5th ed. Washington (DC): American Psychiatric Association; 2013.

2. ZAFEIRIOU DI, VERVERI A, VARGIAMI E. Childhood autism and associated comorbidities. Brain Dev. 2007; 29(5):257-72. Review.

3. CHRISTENSEN DL, BAIO J. et al. Centers for Disease Control and Prevention (CDC). Prevalence and characteristics of autism spectrum disorder among children aged 8 years - Autism and Developmental Disabilities Monitoring Network, 11 Sites, United States, 2012. MMWR Surveill Summ. 2016;65(3):1-23. Erratum in: MMWR Morb Mortal Wkly Rep. 2016;65(15):404.

4. FOMBONNE E. Epidemiology of pervasive developmental disorders. Pediatric Research, 2009; 65(6), 591-598.

5. CANITANO R. Epilepsy in auti sm spectrum disorders. Eur Child Adolesc Psychiatry. 2007; 16:61-6.

6. SPENCE SJ, SCHNEIDER MT. The role of epilepsy and epilepti form EEGs in auti sm spectrum disorders. Pediatr Res. 2009; 65:599-606.

7. Associação Americana de Psiquiatria. Manual Diagnóstico e Estatístico de Transtornos Mentais, $5^{\mathfrak{a}}$ edição (DSM5). Porto Alegre: Artmed; 2014.

8. BRASIL. Instrumentos Diagnósticos para Avaliar o Autismo. INSTITUTO INCLUSÃO BRASIL. Disponível em : http://www.institutoinclusaobrasil.com.br/instrumentos-diagnosticos-para-avaliar-o-autismo/. Acesso em: 06 junhos de 2018.

9. GADIA CA, TUCHMAN, R, ROTTA NT. Autismo e doenças invasivas de desenvolvimento. Jornal de Pediatria Vol. 80, №2 (supl), 2004.

10. BRASIL. Linha de cuidado para a atenção às pessoas com transtornos do espectro do autismo e suas famílias na Rede de Atenção Psicossocial do Sistema Único de Saúde. Secretaria de Atenção à Saúde, Departamento de Atenção Especializada e Temática. - Brasília: Ministério da Saúde, 2015.

11. OLIVEIRA KG, SERTIÉ AL. Transtorno do espectro autista: um guia atualizado para aconselhamento genético. Revista de Ciências Básica. Hospital Israelita Albert Einstein, São Paulo, SP, Brasil. 2017 ; 15 (2): 233-8.

12. KUCZYNSKI E. Detecção precoce de epilepsia Lato sensu: importância diagnóstica e prognóstica nos transtornos do Espectro Autismo. XXXIII Congresso Brasileiro de Psiquiatria da ABP, Florianópolis- SC, Mai/Jun 2016.

13. ZILBOVICIUS M, MERESSE I, BODDAERT N. Autismo: neuroimagem. Rev Bras Psiquiatr. 2006; 28(Supl I):S218.

14. GADIA CA, TUCHMAN R, ROTTA NT. Autismo e doenças invasivas de desenvolvimento. Jornal de Pediatria Vol. 80, №2 (supl), 2004.

15. ZANON RB, BACKES B, BOSA CA. Identificação dos Primeiros Sintomas do Autismo pelos Pais. Universidade Federal do Rio Grande do Sul. Psicologia: Teoria e PesquisaJan-Mar 2014, Vol. 30 n. 1, pp. 25-33.

16. SOUZA VMA, et al. Síndrome de West, Autismo e Displasia Cortical Temporal: Resolução da Epilepsia e Melhora do Autismo com Cirurgia. Programa de Cirurgia de Epilepsia. J Epilepsy Clin Neurophysiol 2008; 14(1):33-37.

17. D'ORSI G. et al. Clinical features and long-term outcome of epilepsy in periventriculae nodular heterotopia. Simple compared with plus forms. J Neurol. Neurosurg. Psychiatry. Jun. 2004; 75(6): 873-878.

18. MUÑOZ-YUNTA JA. et al. Autismo y epilepsia. Acta Neurol Colomb. Jun 2006; 22(2); 112-117.

19. MUÑOZ-YUNTA JA. et al. Clínica de la epilepsia em los transtornos del espectro autista. Rev Neurol. 2003; 36(1); 61-67. 